\section{Focused lon Beam Microscopy and Micromachining}

\section{C.A. Volkert and A.M. Minor, Guest Editors}

\begin{abstract}
The fairly recent availability of commercial focused ion beam (FIB) microscopes has led to rapid development of their applications for materials science. FIB instruments have both imaging and micromachining capabilities at the nanometer-micrometer scale; thus, a broad range of fundamental studies and technological applications have been enhanced or made possible with FIB technology. This introductory article covers the basic FIB instrument and the fundamentals of ion-solid interactions that lead to the many unique FIB capabilities as well as some of the unwanted artifacts associated with FIB instruments. The four topical articles following this introduction give overviews of specific applications of the FIB in materials science, focusing on its particular strengths as a tool for characterization and transmission electron microscopy sample preparation, as well as its potential for ion beam fabrication and prototyping.
\end{abstract}

\section{Introduction}

The focused ion beam (FIB) microscope has gained widespread use in fundamental materials studies and technological applications over the last several years because it offers both high-resolution imaging and flexible micromachining in a single platform.

The FIB instrument is similar to a scanning electron microscope (SEM), except that the beam that is rastered over the sample is an ion beam rather than an electron beam. Secondary electrons are generated by the interaction of the ion beam with the sample surface and can be used to obtain high-spatial-resolution images. In most commercially available systems, $\mathrm{Ga}$ ions are used, and their sputtering action enables precise machining of samples. In conjunction with the gas-injection capabilities on these systems, which enable ion-beam-activated deposition and enhanced etching, a range of sample fabrication schemes are possible.

During the last 25 years, FIB instrumentation has become an important technology for a wide array of materials science applications, from circuit editing and transmission electron microscopy (TEM) sample preparation to microstructural analysis and prototype nanomachining. Most modern FIB instruments supple- ment the FIB column with an additional SEM column so that the instrument becomes a versatile "dual-beam" platform (FIB-SEM, see Figure 1) for imaging, material removal, and deposition at length scales of a few nanometers to hundreds of microns. The FIB instrument becomes a powerful tool for nanomanipulation and fabrication through the augmentation of an FIB instrument with micromanipulators and gas injection for local chemical vapor deposition (CVD).

The first FIB instruments evolved from advances in field ion microscopes ${ }^{1}$ and through the development of highresolution liquid metal ion sources (LMISs) ${ }^{2-4}$ In the 1980s, FIB instruments were embraced by the semiconductor industry as offline equipment for mask or circuit repair. It was not until the 1990s that FIB instruments began to be used in research laboratories, and today there are commercial instruments available from multiple manufacturers. ${ }^{5}$ With the popularity of FIB instruments for TEM sample preparation, microstructural analysis, and nanomachining, dual-beam FIB instruments are becoming a versatile and powerful tool for materials researchers.

This introductory article focuses on the FIB instrument itself and the basic ion-solid interactions that lead to the various functionalities of FIBs. In the topical articles that follow, the major subspecialties of FIB research are discussed.

\section{The FIB Instrument}

The basic functions of the FIB, namely, imaging and sputtering with an ion beam, require a highly focused beam. A consistent tenet of any focused beam is that the smaller the effective source size, the more current that can be focused to a point. Unlike the broad ion beams generated from plasma sources, high-resolution ion beams are defined by the use of a field ionization source with a small effective source size on the order of $5 \mathrm{~nm}$, therefore enabling the beam to be tightly focused.

The ion source type used in all commercial systems and in the majority of research systems designed with micromachining applications in mind is the liquidmetal ion source (LMIS). ${ }^{6,7}$ Of the existing ion source types, the LMIS provides the brightest and most highly focused beam (when connected to the appropriate optics). There are a number of different types of LMIS sources, the most widely used being a Ga-based blunt needle source. Ga has decided advantages over other LMIS metals such as In, $\mathrm{Bi}, \mathrm{Sn}$, and $\mathrm{Au}$ because of its combination of low melting temperature $\left(30^{\circ} \mathrm{C}\right)$, low volatility, and low vapor pressure. The low melting temperature makes the source easy to design and operate, and because Ga does not react with the material defining the needle (typically W) and evaporation is negligible, Ga-based LMISs are typically more stable than other LMIS metals. During operation, Ga flows from a reservoir to the needle tip (with an end radius of about $10 \mu \mathrm{m}$ ), where it is extracted by field emission. A large negative potential between the needle and an extraction electrode generates an electric field of magnitude $10^{10} \mathrm{~V} / \mathrm{m}$ at the needle tip. The balance between the electrostatic forces and the $\mathrm{Ga}$ surface tension wetting the tapered $\mathrm{W}$ needle geometry results in the formation of a single Taylor cone at the needle tip. For typical emission currents used in FIB microscopes $(\sim 2 \mu \mathrm{A})$, a cusp forms at the tip of the Taylor cone with a tip radius of approximately $5 \mathrm{~nm}$.

The simplest and most widely used ion beam columns consist of two lenses (a condenser and objective lens) to define the beam and then focus it on the sample, beam-defining apertures to select the beam diameter and current, deflection plates to raster the beam over the sample surface, stigmation poles to ensure a spherical beam profile, and a high-speed beam blanker to quickly deflect the beam 


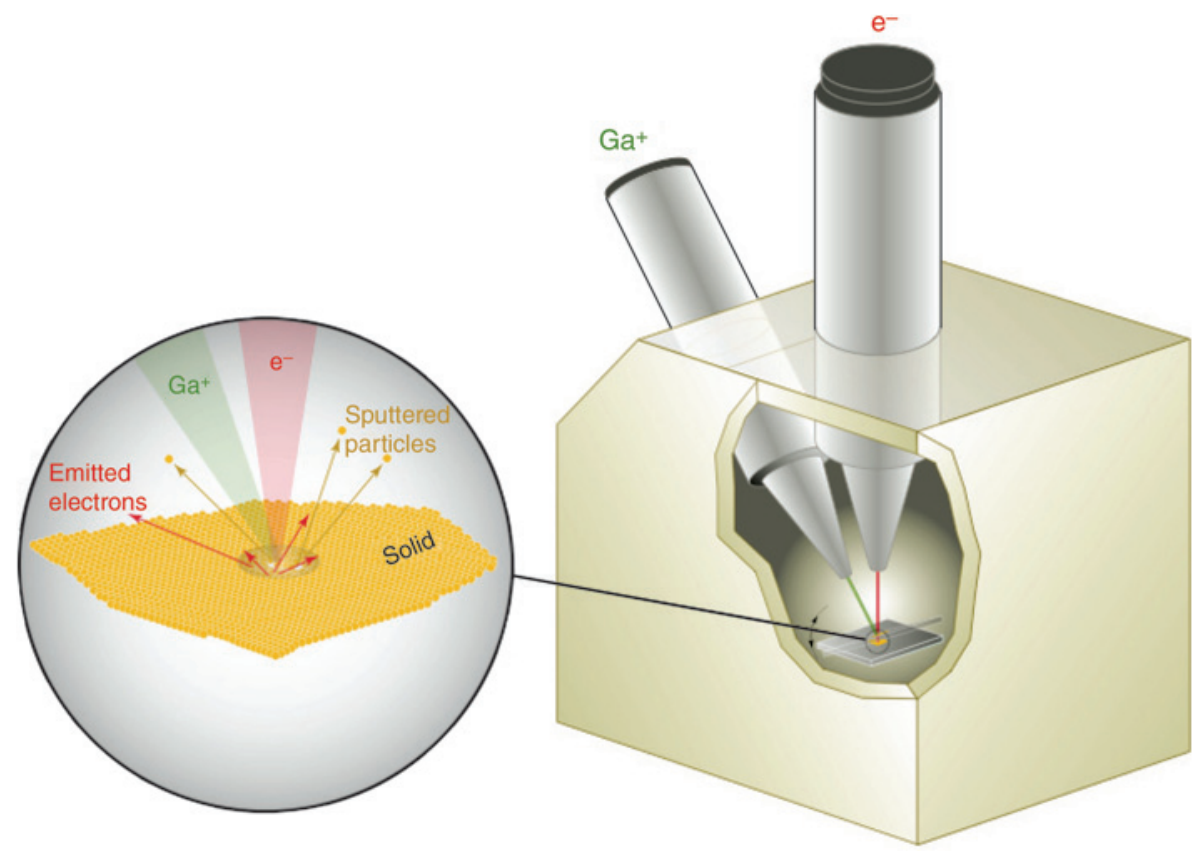

Figure 1. Schematic illustration of a dual-beam FIB-SEM instrument. Expanded view shows the electron and ion beam sample interaction.

off the sample and onto a beam stop such as a Faraday cup. Because the focusing strength of an electromagnetic lens is directly related to the charge/mass ratio of a particle, it is impractical to build electromagnetic lenses for ions (which would weigh thousands of kilograms); thus, focusing and steering are performed using electrostatic components rather than the electromagnetic components used for electrons.

The size and shape of the beam intensity profile on the sample determines the basic imaging resolution and micromachining precision. Generally, the smaller the beam diameter, the better the achievable resolution and milling precision, although the requirements for the two applications are not exactly the same. ${ }^{8}$ For the energies, currents, and acceptance angles used in typical FIB systems, the beam spot size is limited mostly by the chromatic aberration that results primarily from the energy spread of the beam due to space charge effects at the ion source and secondarily from the spherical aberration of the lenses. However, the ultimate spatial resolution for FIB imaging is, in fact, limited by sputtering and is thus sample-dependent. ${ }^{9}$ In modern FIB systems, the imaging resolution determined by the sputter-limited sig$\mathrm{nal} /$ noise usually is about $10 \mathrm{~nm}$.

The sample is mounted on a grounded stage with three-axis translation, rotation, and tilt capabilities. The stage is designed to have a eucentric point (i.e., a wellcentered point such that the field of view is maintained when tilting the specimen) at the location where the two beams cross (or at the working distance of the ion beam, in the case of a single-beam FIB). The region of interest on the sample is moved to the eucentric point using translation and rotation and then tilted for the desired angle of beam incidence. The total current on the sample (sum of the incoming ion or electron beam and all emitted charged particles) is measured at the stage.

Depending on the application, the various emitted particles or radiation can be detected with appropriate detectors in the sample chamber. Traditional detectors such as those in an SEM can be used to detect the electrons or x-rays created by the interaction of the ion beam with the sample. The ions sputtered from the sample can also be detected using a variety of detectors such as charge electron multipliers, and mass selection of the sputtered charged particles is also possible (secondary ion mass spectrometry). FIBs derive an important additional functionality through the use of gas-injection sources to deliver gas locally to either enhance the etching rate or result in site-specific CVD. Secondary electrons generated by the incident ion beam (or, alternatively, the incident electron beam in dual-beam systems) can crack hydrocarbon precursor gases, leading to local deposition of the conducting material $(\mathrm{W}, \mathrm{Pt}$, or $\mathrm{C}$ ) or insulating material $\left(\mathrm{SiO}_{2}\right)$; see the article by MoberlyChan et al. in this issue. The local deposition of material also enables sophisticated micromanipulation within the FIB chamber, made possible through micromanipulation accessories and the ability of the FIB to cut (sputter), paste (deposit), and watch (image) during a manipulation process within the chamber. The result is a system that can image, analyze, sputter, and deposit material all with very high spatial resolution and controlled through one software program. In a large part, it is this multifunctional versatility that has made FIB instruments popular among materials researchers.

\section{Ion-Solid Interactions}

Ion-solid interactions play an important role in many different endeavors, ranging from fabrication of microelectronic devices to understanding distributions of cosmic gases. This brief introduction will be limited to the processes and conditions relevant to the use of FIB systems in materials science. For more detailed descriptions of ion-solid interactions, the reader is referred to books and review articles on the subject ${ }^{6,10-12}$ as well as to literature specifically on FIB. ${ }^{13-15}$

When an ion impinges on a solid, it loses kinetic energy through interactions with the sample atoms. This transfer of energy from the ion to the solid results in a number of different processes (see Figure 2):

- ion reflection and backscattering

- electron emission

- electromagnetic radiation

- atomic sputtering and ion emission

- sample damage

- sample heating

The ion typically comes to rest in the solid, leading to implantation of the ion. With the possible exception of electromagnetic radiation generation, all of these processes are important to FIB and FIB-SEM system applications and are described in the next sections.

\section{Collision Cascade}

Ion kinetic energy and momentum are transferred to the solid through both inelastic and elastic interactions. In inelastic interactions (called electronic energy loss), ion energy is lost to the electrons in the sample and results in ionization and the emission of electrons and electromagnetic radiation from the sample. In elastic interactions (called nuclear energy loss), ion energy is transferred as translational energy to screened target atoms and can result in damage (displacement of sample 


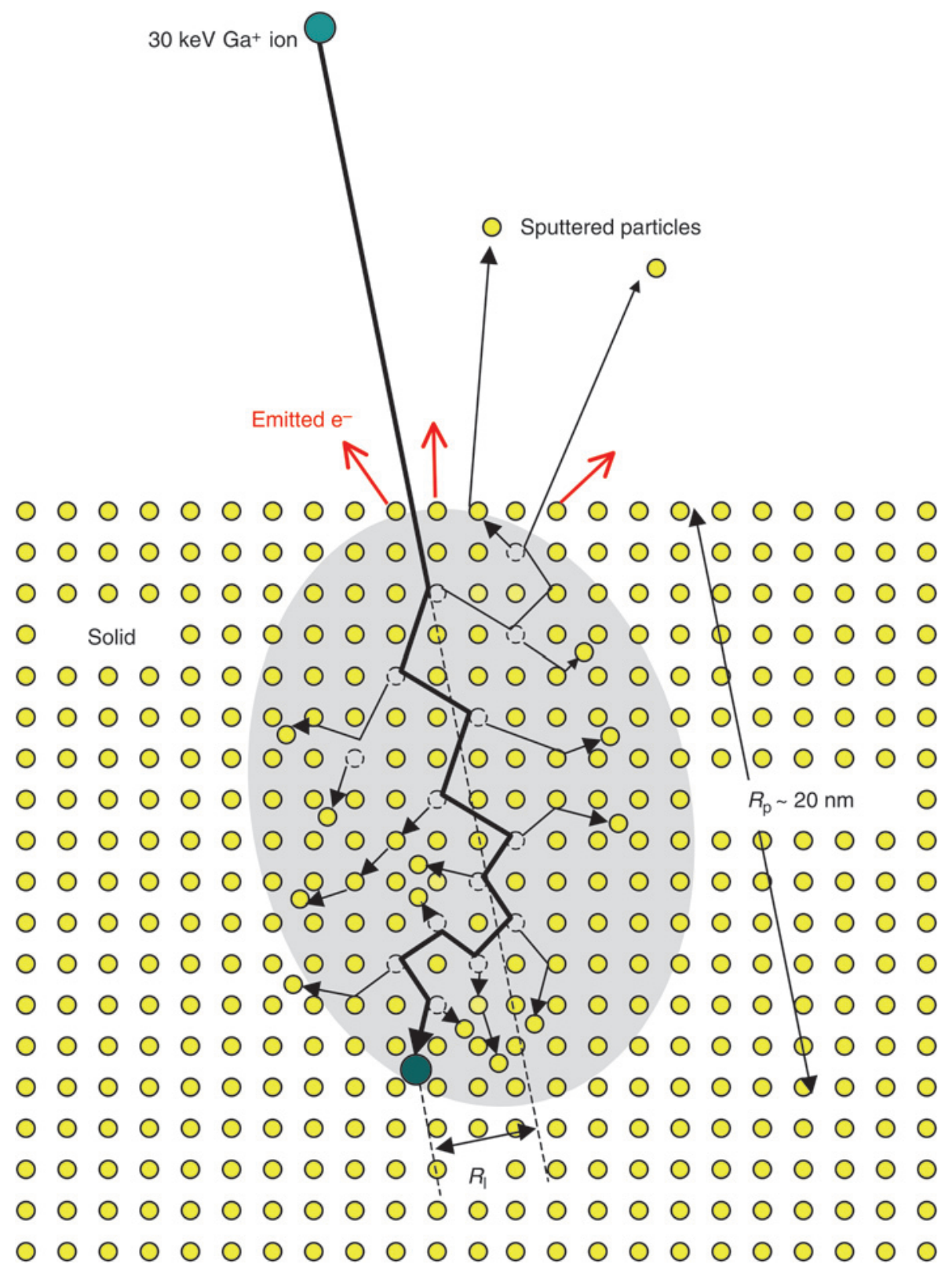

Figure 2. Schematic illustration of a collision cascade generated by a $30 \mathrm{keV} \mathrm{Ga}$ ion incident on a crystal lattice, showing the damage created in the collision cascade volume, and the projected range $R_{\mathrm{p}}$ and lateral range $R_{l}$ of the implanted ion.

atoms from their initial sites) and sputtering from the sample surface.

The most widely accepted concept for ion-solid interactions is the collision cascade model ${ }^{16,17}$ (Figure 2). For the case of 5-30 keV Ga impinging on most solids, the collision cascade involves a series of independent binary collisions (the linear collision cascade regime). If the translational energy transferred to a target atom during a collision exceeds a critical value called the displacement energy, the atom will be knocked out of its original site, for the collisions are nonadiabatic, because of the very short time scale.

After approximately $10^{-11} \mathrm{~s}$, the 5-30 $\mathrm{keV} \mathrm{Ga}$ ion comes to rest in the solid, and the energies of all particles participating in the cascade have decreased below the displacement energy. At this point, the collision cascade has ended. What remains are the emitted particles and radiation, and ion beam damage such as lattice defects, incorporated $\mathrm{Ga}$, and heat, all of which may continue to interact and evolve.

Molecular dynamics calculations are ideally suited for simulating collision cascades, because of the short length and time scales. Monte Carlo calculations are also well suited to simulating ion-solid interactions by including the "frictional" electronic stopping and stochastic elastic collisions. The most widely used Monte Carlo simulation is the program TRIM or SRIM (transport, or stopping range, of ions in matter). ${ }^{18}$ Such calculations for 30 $\mathrm{keV} \mathrm{Ga}$ into elements from $\mathrm{Li}$ to Bi show that roughly two times as much of the ion energy is lost to nuclear energy losses than to ionization energy losses (the former from interactions with the nucleus of an atom and the latter from interactions with the electrons). Of the nuclear energy losses, the majority is lost through sample atom vibrations or heating rather than through vacancy formation.

The projected and lateral ranges of the $30 \mathrm{keV} \mathrm{Ga}$ scale inversely with the sample density and are between $10 \mathrm{~nm}$ and 100 $\mathrm{nm}$ (projected) and between $5 \mathrm{~nm}$ and 50 nm (lateral). TRIM sputtering yields (sputtered target atoms per incoming ion) are between 1 and 20 for normalincidence $30 \mathrm{keV} \mathrm{Ga}$ and increase somewhat with atomic number. ${ }^{6}$ However, sputtering yield predictions depend critically on surface binding energies, which are not well known and are sensitive to surface structure and chemistry. TRIM vacancy generation predictions between 300 and 1000 vacancies per incoming ion are overestimates because defect diffusion and interactions are ignored. In addition, discrepancies between experimental and simulated ranges and collision cascade shapes in crystals can be expected, because TRIM samples are isotropic and cannot capture channeling effects. Despite these limitations, such calculations are invaluable in predicting trends and in estimating the effects of ion-solid interactions.

\section{Ion Beam Imaging}

In the same manner that images are generated in an SEM, the ion beam can be rastered over a sample surface and the emitted electrons, particles (atoms and 
ions), and electromagnetic radiation can be detected. Conventional SEM imaging is based on detecting the secondary electrons (SEs). To date, most imaging in a FIB is based on detecting the low-energy electrons, often referred to as ion-induced secondary electrons (ISEs). Typically, 1-10 electrons with energies below $10 \mathrm{eV}$ are generated per incoming 5-30 keV Ga ion. These electrons are created by both kinetic and potential emission from the top few atomic layers where the primary ion impacts the solid as well as where backscattered or sputtered particles exit the sample. The total low-energy electron yield depends strongly on surface oxidation and contamination and thus will change as the surface is sputter-cleaned and $\mathrm{Ga}$ is incorporated.

Ion beams are not as finely focused as electron beams and, partly for this reason, they generally offer lower resolution. However, the contrast mechanisms for ISE generation are different from those for SE generation and can offer complementary information about a sample surface. SE and ISE images of the same sample are shown in Figure 3. Both the SE (Figure 3a) and the ISE (Figure 3b) images show contrast due to surface topography and material differences. However, ISE imaging typically delivers stronger channeling contrast from crystals than SE imaging. The contrast due to crystal orientation is easily distinguished from material contrast, because crystal contrast changes with the incidence angle of the ion beam and material contrast does not. In ideal samples such as $\mathrm{Cu}$ or $\mathrm{Au}$, ISE channeling contrast can reveal twin lamellae as narrow as 20 $\mathrm{nm}$ and grains as small as $50 \mathrm{~nm}$.

The different contrast mechanisms are illustrated in Figure 4. A comparison of Figures $4 \mathrm{a}$ and $4 \mathrm{~b}$ shows that when the crystal is oriented so that the ion "channels" along crystal planes, there are fewer ion interactions with sample atoms near the surface and thus fewer electrons are emitted. Figure 4c illustrates that heavier samples typically result in more ISEs (and SEs). Figure $4 \mathrm{~d}$ shows that surface topography can lead to increases in the number of ISEs (and SEs), because of the increase in the number of ion-solid interactions near the sample surface. The SE and ISE images are also often distinguished by the amount of charging generated in insulating samples.

Presumably because of differences in the low-energy/secondary electron yields and to the fact that the Ga implantation creates a thin conducting layer at the sample surface, the FIB can often be used to image uncoated samples that are difficult to image even with low-voltage SEM. However, it is important to remember that ion beam imaging always results in some Ga implantation and sputtering of the sample surface.
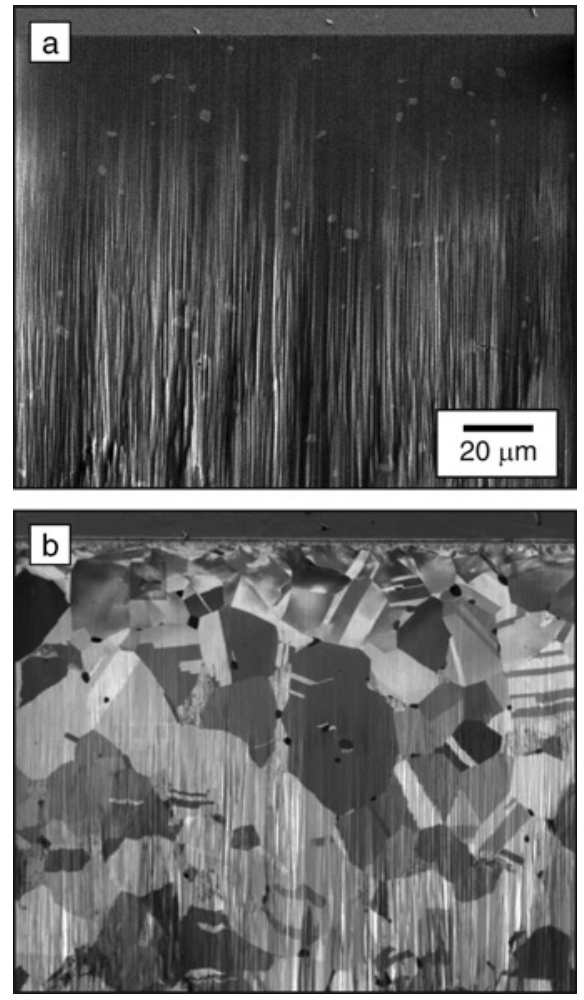

Figure 3. (a) Secondary electron (SE) and (b) ion-induced secondary electron (ISE) images of an FIB-cut cross section in brass. Surface topography generated during the FIB milling and phase contrast are visible in both images. The heavier second-phase precipitates are bright in the SE image and dark in the ISE image. ${ }^{37}$ Channeling contrast showing the grain structure is visible only in the ISE image.

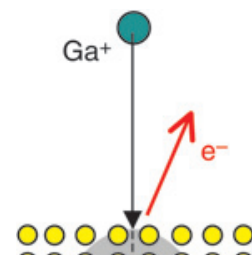

0000 O० 000 000010000 000 O:० 000 000 an 000 00000000 00000000 00000000 a

\section{Ion Beam Sputtering}

Because of the sputtering action of the ion beam, the FIB can be used to locally remove or mill away material. For example, a Sn sphere is progressively sputtered over a defined area in Figure 5. For direct milling, the limiting feature size is typically about $10 \mathrm{~nm}^{9,19,20}$ (see the articles by MoberlyChan et al. and Langford et al. in this issue). Quantitative aspects of sputtering are complicated and depend on the material, crystal orientation, ion beam incidence angle, and the extent of redeposition.

As the incidence angle of the ion beam is increased, the intersection of the collision cascade with the sample surface increases, and the number of sputtered atoms per collision cascade increases (a similar effect of geometry on electron emission is shown in Figure $4 \mathrm{~d}$ ). However, at the same time, the fraction of reflected or backscattered $\mathrm{Ga}$ ions increases. The combination of these two effects leads to a maximum in sputtering yield at an incidence angle of approximately $75-80^{\circ}$. This effect has been confirmed for $25-30 \mathrm{keV}$ Ga into a variety of materials, including single-crystal $\mathrm{Si}_{1}{ }^{19-22}$ amorphous $\mathrm{SiO}_{2}, 21,22$ and polycrystalline $\mathrm{Au}$ and $\mathrm{W}^{21}$ and shows good agreement between experiment and theory. Si or amorphous solids are ideal for such a study because the effects of crystal channeling are avoided (the surface region of $\mathrm{Si}$ amorphizes under the $\mathrm{Ga}$ beam $^{7}$ ). The behavior is more complicated in crystalline material where both incident angle and channeling effects are present. ${ }^{21}$

The sputtering yield at a given incidence angle can vary by as much as a factor of 10 for strongly channeling crystal orientations in materials such as $\mathrm{Cu}^{23}$ This is because for easy channeling orienta-

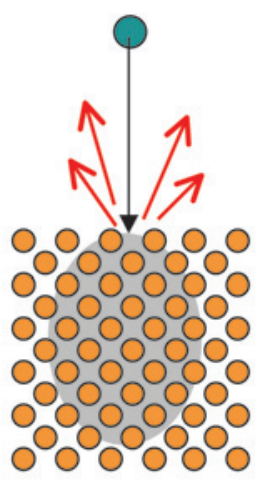

c

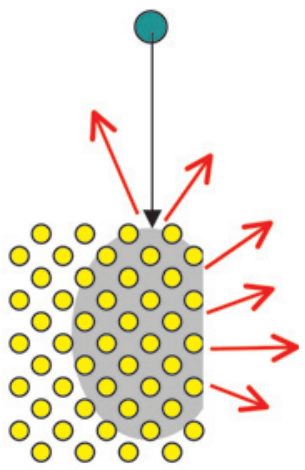

d

Figure 4. Schematics showing the influence of (a), (b) crystal orientation, (c) atomic mass, and (d) surface geometry on $30 \mathrm{keV} \mathrm{Ga}^{+}$collision cascades and ISE image contrast formation. The orange atoms in (c) are more massive than the yellow atoms in (a), (b), and (d). Similar concepts influence sputtering yields. 
tions, the ion experiences only inelastic glancing-angle collisions with the atoms lying in a crystal plane and travels deeper into the crystal before causing elastic collisions, so that fewer atoms are sputtered from the surface. This is analogous to the effect of crystal orientation on low-energy electron yields illustrated in Figures $4 a$ and $4 \mathrm{~b}$. Channeling effects on sputtering at a vertical grain boundary in $\mathrm{Cu}$ are shown in Figure 6. The grain with the smaller electron yield appears dark in Figure 6. Its lower sputter yield results in a shallower sputter profile than that observed for the brighter neighboring grain.

The sputter profiles also depend on the exact sequence in which the ion beam is
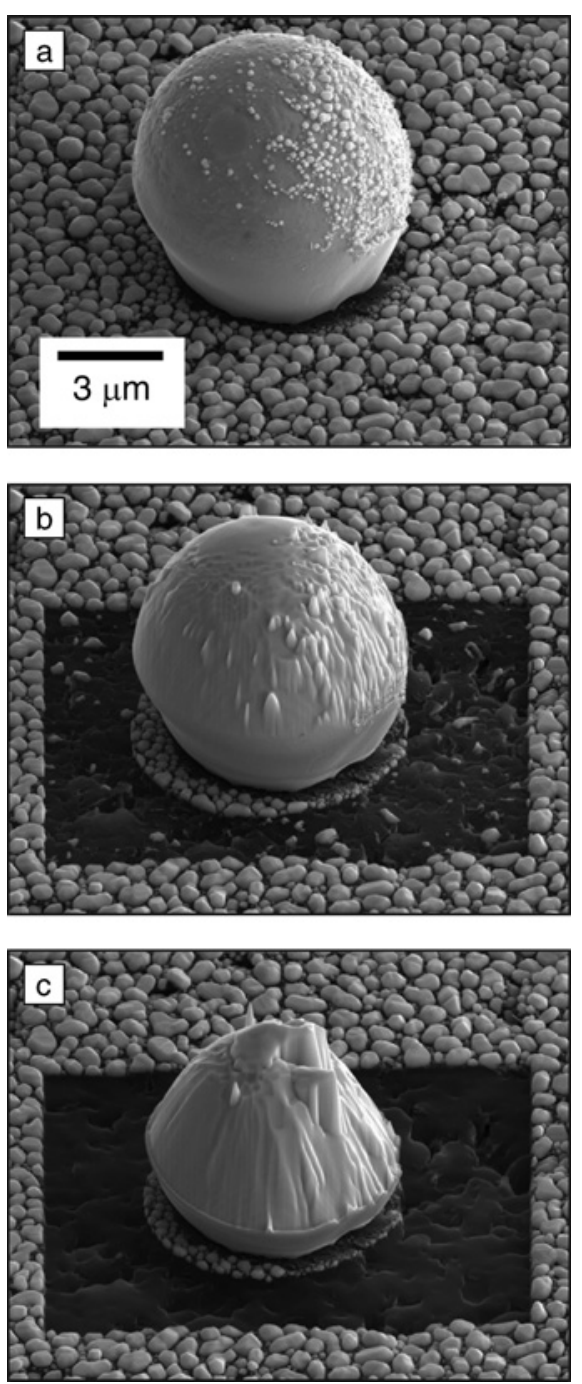

Figure 5. (a)-(c) Secondary electron images during sputtering of a large $\mathrm{Sn}$ sphere surrounded by $\mathrm{Sn}$ droplets, using a $30 \mathrm{keV} \mathrm{Ga}$ beam rastered across a $10 \mu \mathrm{m} \times 10 \mu \mathrm{m}$ area. rastered over the surface. ${ }^{22}$ For instance, the sputter profile of a ring cut by rapid and repetitive scanning ("multi-pass" scanning) differs from that obtained by slowly scanning the beam just once over the same area ("single-pass" scanning) (Figure 6). Despite an identical total ion dose (ions per unit area) for both rings in Figure 6, the slow single-pass scan, spiraling from outside to inside, results in a deeper cut, because of effects of ion focusing, incident angle effects, and redeposition.

Redeposition decreases the effective sputter yield and changes sputter profiles. The decreased yield comes about because redeposited material lands in the area being sputtered and must be sputtered a second time. Redeposition is also given as a reason why completely vertical sidewalls cannot be cut with the FIB without over-tilting the sample, ${ }^{13,24}$ but it is certainly also partly because of the intensity tails of the ion beam profile and of the decrease in sputter yield at high incidence angles. ${ }^{19,20,25}$ Many details of redeposition effects remain open, such as the development of crystal orientation and channeling effects seen in the redeposited material (e.g., Figure 6, single-pass ring).

In addition to redeposition, surface roughening and shadowing effects are prevalent during sputtering. An example of a shadowing effect on topology during cross-sectioning with the FIB, the so-called "curtain effect,"13 is seen in the lower half of the images in Figure 3. Surface roughening, specifically ripple formation, is widespread during ion bombardment ${ }^{9,26}$ and is attributed to competition between smoothing by surface diffusion or viscous flow and roughening because of surfacecurvature-dependent sputter yields. (The sputter yield depends on local curvature for the same reasons it depends on angle of incidence.)

Even during normal-incidence sputtering, surface roughening can occur and is dependent on the crystal orientation, as shown in the multi-pass ring cut across two differently oriented grains in Figure 6. Such crystal-orientation-dependent rippling is attributed to anisotropic surface diffusion. ${ }^{27}$ Other unusual effects of sputtering and redeposition on surface evolution continue to be discovered. ${ }^{28,29}$ Nonetheless, progress is being made toward models that can predict surface evolution during sputtering and can be used to achieve desired sputter profiles (see the article by Langford et al. in this issue).

\section{Ga Incorporation}

Imaging and milling with $\mathrm{Ga}$ ions always result in $\mathrm{Ga}$ incorporation near the sample surface. As the sample surface is sputtered away at a rate proportional to the sputtering yield and the ion flux (ions per area per time), the $\mathrm{Ga}$ is implanted

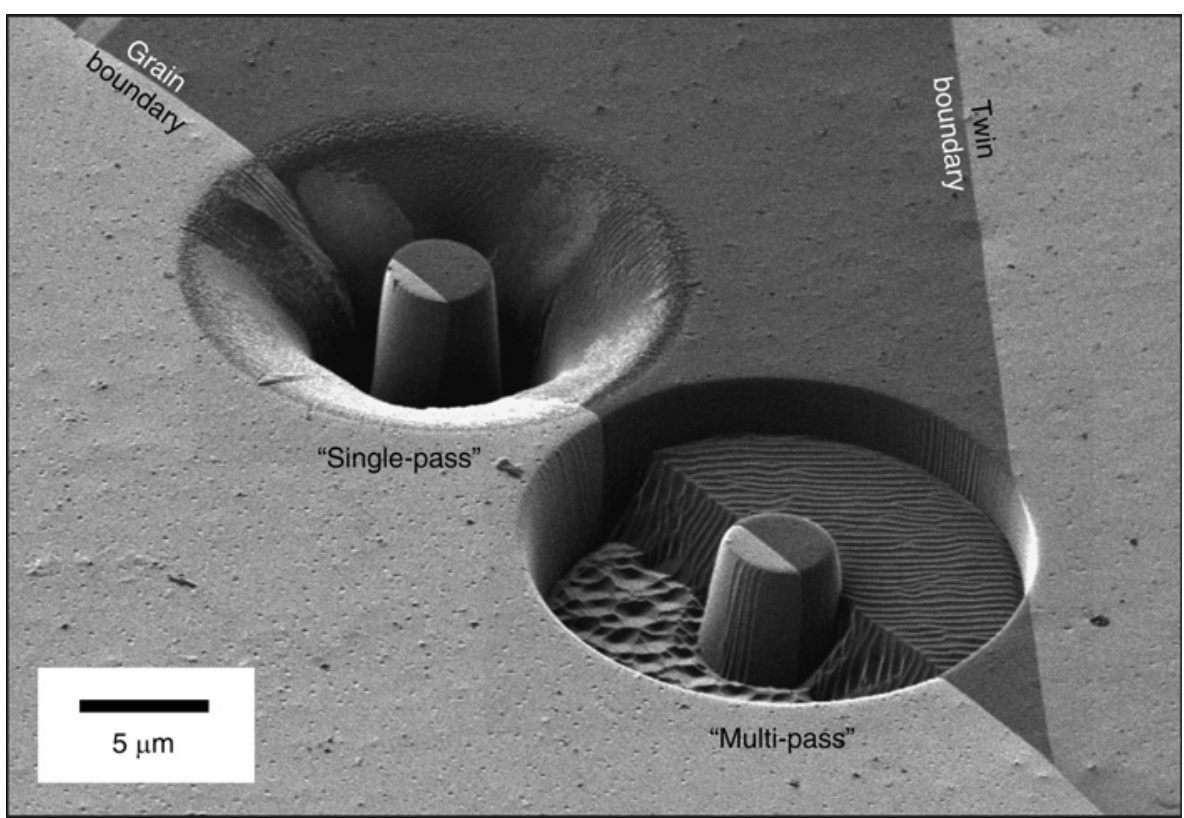

Figure 6. Ion-induced secondary electron image of rings milled with $30 \mathrm{keV} \mathrm{Ga}$ at a grain boundary in $\mathrm{Cu}$. The single-pass ring shows an enhanced sputtering yield (deeper trough) and redeposition (sloped, thicker sidewalls); the multi-pass ring shows channeling effects and surface roughening. 
further into the sample, and a steady-state profile of $\mathrm{Ga}$ is reached. The maximum $\mathrm{Ga}$ concentration occurs at steady state after the removal of target material to a depth roughly equal to the ion range and is constant over a depth also roughly equal to the ion range. Ignoring the effects from possible diffusion of $\mathrm{Ga}$ in the target material, differences in molar volume, and preferential sputtering, the Ga atom fraction at steady state is

$$
f_{\mathrm{Ga}}=1 /(\alpha Y),
$$

where $\alpha$ is the fraction of ions that are not reflected or backscattered from the sample surface, $Y$ is the sputtering yield (number of sputtered atoms/ions per incoming ion) ${ }^{10}$ and $\alpha Y$ is the sputtering yield per implanted ion. Based on this equation and typical TRIM sputtering yields (from 1 to 20 sputtered atoms per incoming ion), atom fractions from 1 at. $\%$ to 50 at. $\%$ Ga are expected near the sample surface. Because of the increase in sputter yield with incident angle, the steady-state $\mathrm{Ga}$ concentration is expected to be roughly 5-10 times smaller during glancing-angle sputtering than normalincidence sputtering. In contrast, channeling is expected to raise the steady-state $\mathrm{Ga}$ concentration.

Most studies report $\mathrm{Ga}$ concentrations in good agreement with simulations and theory, although a few claim to see almost no Ga. Unless the Ga diffuses away or is reflected, it must end up in the surface region of the sample. Some studies were performed with energy-dispersive spectroscopy in an SEM, which for typical electron beam parameters will look through the thin Ga-doped layer at the surface and underestimate the actual Ga concentration. Other studies have used surface Auger analysis (without sputtering) that may also underestimate the Ga concentration, because of the presence of carbonaceous or oxide surface layers. Carbon-based surface layers are particularly prevalent in samples that have been imaged in the SEM after FIB milling. ${ }^{30}$ In contrast, Auger depth profiles or chemical analysis in the TEM give reasonable agreement with predictions for both $\mathrm{Ga}$ concentration and range (allowing for channeling effects).

All of these considerations become much more complicated in alloys or if $\mathrm{Ga}$ diffusion or reactions take place. One unusual example is the formation of Gacontaining surface phases during imaging of certain fcc metals (see the article by Mayer et al. in this issue). This phenomenon, which is coupled with sample crystal structure and channeling effects, limits the ability to obtain high-quality images from certain materials.

\section{Ion Beam Damage}

A major drawback of FIB imaging and machining, particularly for TEM samples, is the damage created by the ion beam (again, see Mayer et al. in this issue). As the ion dose increases, the individual disordered cascade regions overlap and a damaged surface layer is formed. Depending in particular on the sample material and temperature, the ion beam damage can take the form of sample surface amorphization, point defect creation, dislocation formation, phase formation, grain modification, or other unusual effects. With the exception of Si amorphization, systematic investigations of FIB damage are just beginning. Nonetheless, several trends can be identified based on literature results for broad beam ions and on anecdotal FIB observations.

Ion beam amorphization is a wellknown phenomenon and has been extensively studied for covalently bound materials such as $\mathrm{Si}, \mathrm{Ge}, \mathrm{GaAs}$, and $\mathrm{C}$ (diamond). Because of the highly directional nature of the atomic bonds in covalent materials and in certain alloys, the atomic rearrangements necessary to heal the disorder created by the ion beam are often hindered. In contrast, pure metals have nondirectional bonding and do not amorphize. Thus, thin amorphous layers sometimes observed at the edge of pure metal TEM samples made by FIB presumably contain impurities such as $\mathrm{Ga}, \mathrm{C}$, or O. Some oxygen gets in because of the relatively poor quality of the vacuum.

Point defects and dislocation loops can also be created during FIB imaging and machining. Systematic studies of FIBinduced defects have not been undertaken, although there are many isolated observations, both published and unpublished. For example, $\mathrm{Cu}$ is prone to extensive FIB damage, ${ }^{31}$ as shown in the TEM image in Figure 7, but $\mathrm{Al}$ is not and even provides reasonable-quality highresolution TEM samples. ${ }^{32}$ Tooth enamel (hydroxyapatite) is resistant to FIBinduced damage, ${ }^{33}$ but other apatite crystals that decompose at lower temperatures amorphize easily. Several additional and unusual types of damage have been observed in FIB-milled samples. These include the formation of Gacontaining surface phases (see Mayer et al., this issue) as well as ion-beaminduced grain growth in fine-grained $\mathrm{Ni}$ and Ni alloys. ${ }^{34}$ Preferential sputtering, which is prevalent during FIB milling of materials that decompose at low tempera- a

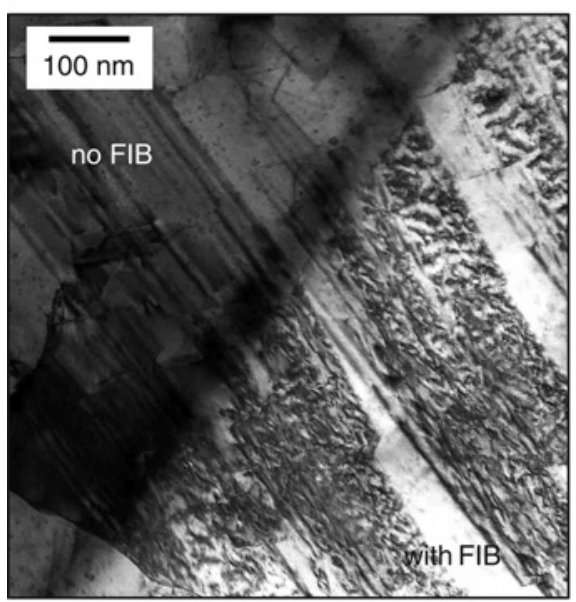

b

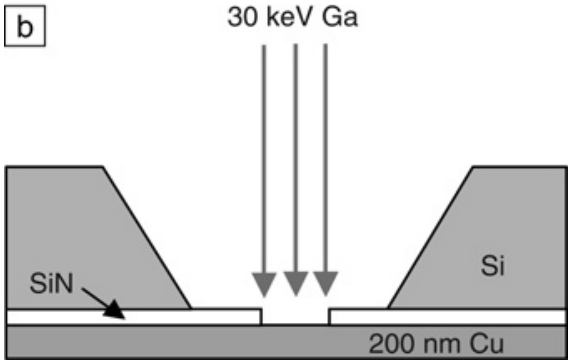

Figure 7. (a) Transmission electron microscopy (TEM) image of a Cu film, showing that the existing dislocation structure was modified by FIB milling (b) Schematic illustration of the milling geometry. (TEM image courtesy of G.P. Zhang.)

tures, can lead to chemical changes in the surface region and influence the ease of damage formation and amorphization.

\section{Ion Beam Heating}

During ion implantation, almost all of the ion kinetic energy is eventually converted to heat, with only a small fraction stored as defects in the sample or emitted as energetic particles or radiation. ${ }^{14}$ For times longer than approximately a nanosecond and distances larger than around $100 \mathrm{~nm}$, the ion beam can be approximated as a continuous heat source. At shorter times, there are large temporal variations in heating, and at times of less than $10^{-12} \mathrm{~s}$, the atoms barely have time to interact with each other, and the temperature of the solid is not well defined. ${ }^{15}$ The maximum temperature reached in a sample depends on the beam power $P$, sample thermal conductivity $\kappa$, sample geometry, and contact to a heat reservoir. ${ }^{15,35}$ Beam powers in commercial systems have maximum values of $1 \mathrm{~mW}$. 
When the ion beam is incident on a flat surface, the transfer of heat away from the incidence point is so effective that even in the absence of a heat reservoir (e.g., for a semi-infinite sample), a finite steady-state temperature increase is reached given by ${ }^{36}$

$$
T=P /(\pi a \kappa),
$$

where $a$ is the radius of the circular ion beam profile on the sample surface. For values of $P / a$ available in commercial FIBs, between $1 \mathrm{~W} / \mathrm{m}$ and $1000 \mathrm{~W} / \mathrm{m}$, the temperature rise predicted by Equation 2 can range from entirely negligible for samples with good thermal conductivity to huge for poor conductors. For example, for $\mathrm{Si}(\kappa=148 \mathrm{~W} / \mathrm{m} \mathrm{K})$ the temperature increase is $<2^{\circ} \mathrm{C}$ even for the most extreme beam conditions. In contrast, for polymeric or biological materials $(\kappa \sim 0.1 \mathrm{~W} / \mathrm{m}$ $\mathrm{K})$, such small temperature rises are only achieved with $P / a$ values of $<2 \mathrm{~W} / \mathrm{m}$, which is at the low end of what is available in commercial FIBs.

Beam heating can be diminished by placing samples in good contact with a heat reservoir. On the other hand, when imaging or machining TEM lamellae, membranes, or other structured samples, much higher temperatures may be reached when the sample geometry limits the transfer of heat. ${ }^{35}$ An extreme example of this is when the heat transfer is reduced to one dimension, such as for a cylindrical pillar with a diameter equal to the beam diameter. In this case, the temperature rise is given by Equation 2 multiplied by the height-to-diameter ratio of the pillar. Thus, by determining the length-to-width ratio of the thermal path in a structured sample, a worst-case estimate of the actual temperature rise can be obtained and used to select appropriate beam conditions. However, imaging or cutting of highaspect-ratio features in low-conductivity materials may lead to unacceptably high temperatures, even for the most mild beam conditions in commercial systems.

\section{In This Issue}

Four articles follow this introduction and are intended to cover the most widely used FIB applications as well as the current state of the art in FIB materials research. In the first article, by Mayer et al., one of the most important applications of the FIB is presented. The FIB not only enables the preparation of sitespecific samples of uniform thickness, but it also facilitates the fabrication of lamellae from composite samples consisting of materials with very different properties. The strengths of the method as well as the problems, such as FIB-induced damage and Ga contamination, are illustrated with examples. The second article, by Uchic et al., describes how the FIB can be used as a tool for three-dimensional characterization by complementing 2D imaging or mapping with serial sectioning at a submicron level. The third article, by Langford et al., is organized according to the general technological area, such as microelectronics, photonics, microelectromechanical systems, and rapid prototyping. The strengths and limitations of FIB micro- or nanostructuring are illustrated through representative examples, and the article ends with a brief summary and a look to the future of FIB nanostructuring. In the fourth and final article, by MoberlyChan et al., advanced topics such as single-ion implantation, surface morphology during ion-induced erosion, and ion-induced CVD are discussed.

We hope that this issue of MRS Bulletin will inform readers about the fundamental science and current applications of focused ion beam microscopy and micromachining and give them a sense of the future potential of FIB in materials science.

\section{Acknowledgments}

A.M. Minor was supported by the Director, Office of Science, Office of Basic Energy Sciences, of the U.S. Department of Energy under contract DE-AC0205CH11231.

\section{References}

1. E.W. Mueller, T.T. Tsong, Field Ion Microscopy Principles and Applications (American Elsevier, New York, 1969).

2. V.E. Krohn, G.R. Ringo, Appl. Phys. Lett. 27, 479 (1975).

3. R.L. Seliger, J.W. Ward, V. Wang, R.L. Kubena, Appl. Phys. Lett. 34, 310 (1979).

4. L.W. Swanson, Nucl. Instrum. Methods Phys. Res., Sect. 218, 347 (1983).

5. For example, FEI Co., Carl Zeiss Inc., Seiko Instruments Inc., Hitachi Inc., JEOL Ltd., Orsay Physics.

6. A. Benninghoven, F.G. Rüdenauer, H.W Werner, Secondary Ion Mass Spectrometry: Basic Concepts, Instrumental Aspects, Applications and Trends (John Wiley, New York, 1987)

7. P.D. Prewitt, G.L.R. Mair, Focused Ion Beams from Liquid Metal Ion Sources (John Wiley, New York, 1987) p. 291.

8. J. Orloff, Rev. Sci. Instrum. 64, 1105 (1993).

9. J. Orloff, L.W. Swanson, M. Utlaut, J. Vac. Sci. Technol., B 14, 3759 (1996).
10. M. Nastasi, J.W. Mayer, J.K. Hirvonen, IonSolid Interactions: Fundamentals and Applications (Cambridge University Press, Cambridge, UK, 1996).

11. E. Chason et al., Appl. Phys. Rev. 81, 6514 (1997).

12. J.S. Williams, J.M. Poate, Ion Implantation and Beam Processing (Academic, Sydney, 1984).

13. J. Orloff, M. Utlaut, L. Swanson, High Resolution Focused Ion Beams: FIB and its Applications (Kluwer Academic, Dordrecht, 2002).

14. L.A Giannuzzi, F.A. Stevie, Introduction to Focused Ion Beams: Instrumentation, Theory, Techniques, and Practice (Springer, New York, 2005).

15. J. Melngailis, J. Vac. Sci. Technol., B 5, 469 (1987).

16. P. Sigmond, Phys. Rev. 184, 383 (1969).

17. P. Sigmond, I. Mater. Sci., 8, 1545 (1973)

18. J.F. Ziegler, J.P. Biersack, U. Littmark, The Stopping Range of Ions in Solids (Pergamon Press, New York, 1984). The SRIM code is available online at www.srim.org (accessed February 2007).

19. C. Lehrer, L. Frey, S. Petersen, H. Ryssel, J. Vac. Sci. Technol., B 19, 2533 (2001).

20. L. Frey, C. Lehrer, H. Ryssel, Appl. Phys. A 76, 1017 (2003).

21. X. Xu, A.D. Della Ratta, J. Sosonkina, J. Melngailis, J. Vac. Sci. Technol., B 10, 2675 (1992)

22. D. Santamore, K. Edinger, J. Orloff, J. Melngailis, J. Vac. Sci. Technol., B 15, 2346 (1997)

23. B.W. Kempshall et al., I. Vac. Sci. Technol., B 19, 749 (2001).

24. B.I. Prenitzer et al., Metall. Trans. A 29, 2399 (1998)

25. T. Ishitani, H. Tsuboi, T. Yaguchi, H. Koike, J. Electron Microsc. 43, 322 (1994).

26. E. Chason, M.J. Aziz, Scripta Mater. 49, 953 (2003)

27. H.X. Qian et al., Appl. Surf. Sci. 240, 140 (2005).

28. H.H. Chen et al., Science 310, 294 (2005).

29. M. Castro, R. Cuerno, L. Vázquez, R. Gago, Phys. Rev. Lett. 94, 016102 (2005).

30. C.A. Volkert, E.T. Lilleodden, Philos. Mag 86, 5567 (2006)

31. J. Marien, J. Plitzko, R. Spolenak, R. Keller, J. Mayer, J. Microscopy 194, 71 (1999).

32. W. Sigle, private communication (2002)

33. C.A. Volkert, S. Busch, B. Heiland, G. Dehm, J. Microscopy 214, 208 (2004).

34. C.M. Park, J.A. Bain, J. Appl. Phys. 91, 6380 (2002)

35. T. Ishitani, H. Kaga, J. Electron Microsc. 44, 331 (1995).

36. H.S. Carlslaw, J.C. Jaeger, Conduction of Heat in Solids (Oxford University Press, Oxford, UK, ed. 2, 1959) p. 264.

37. T. Ishitani, Y. Madokoro, M. Nakagawa, K. Ohya, J. Electron Microsc. 51, 207 (2002). 


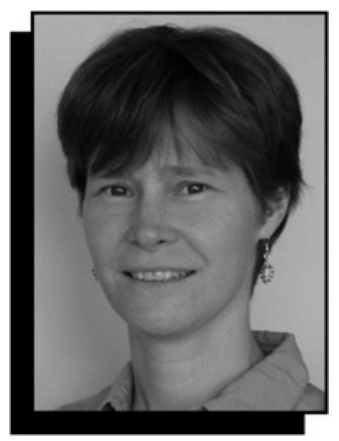

Cynthia A. Volkert

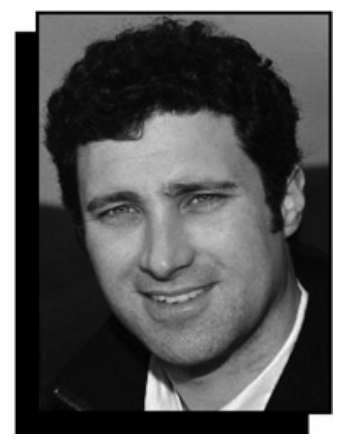

Andrew M. Minor

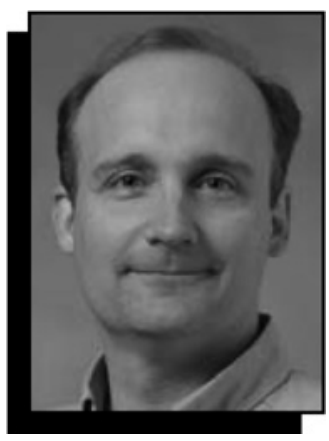

David P. Adams

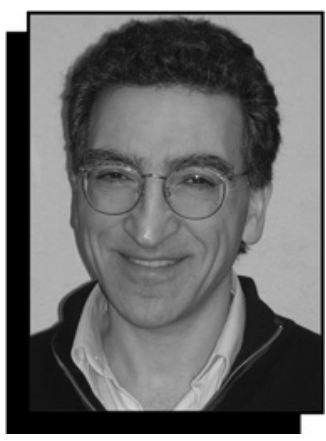

Michael J. Aziz

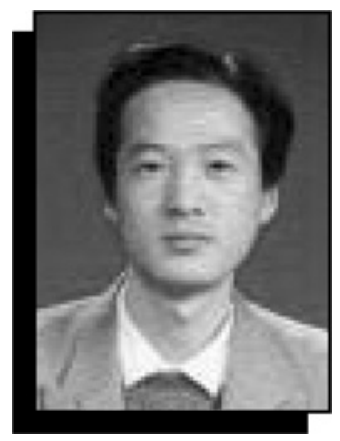

Yongqi Fu
Cynthia A. Volkert, Guest Editor for this issue of MRS Bulletin, is a research scientist and group leader at Forschungszentrum Karlsruhe in Germany, where she specializes in microstructure and mechanical properties studies of small metal structures. She received a bachelor's degree in physics from McGill University and her PhD degree from Harvard University. Volkert then spent 10 years working as a staff scientist at Bell Laboratories before moving to Germany, first to the Max Planck Institute for Metals Research in Stuttgart and then to Karlsruhe.

Volkert can be reached at Institut für

Materialforschung II, Forschungszentrum Karlsruhe, Postfach 36 40 76021, Karlsruhe, Germany; tel. 49-724782-3577, fax 49-424782-2347, and e-mail cynthia.volkert@imf. fzk.de.

Andrew M. Minor, Guest Editor for this issue of MRS Bulletin, is a staff scientist and principal investigator at the National Center for Electron Microscopy at Lawrence Berkeley National Laboratory in Berkeley, California. He received a bachelor's degree in economics and mechanical engineering from Yale University and MS and PhD degrees in materials science and engineering from the University of California, Berkeley. His research group focuses on the mechanical properties of small volumes, in situ TEM technique development, and sample manipulation and preparation methods for electron microscopy investigations of both hard and soft materials.

Minor can be reached at Lawrence Berkeley National Laboratory, 1 Cyclotron Rd., MS 72, Berkeley, CA 94720 USA; tel. 510-495-2749, fax 510486-5888, and e-mail aminor@lbl.gov.

David P. Adams is a distinguished member of technical staff at Sandia National Laboratories. Adams received his bachelor's degree in physics from the University of Virginia and his PhD degree in materials science and engineering from the University of Michigan.

Throughout his technical career, Adams has been involved with thinfilm deposition, studies of microstructure and morphology evolution during film growth, and materials analysis. His current activities involve focused ion beams, including rapid prototyping, development of in situ metrol- ogy techniques, investigations of surface morphology during ion irradiation, and surface micromachining. He has authored or co-authored more than 50 publications and is a member of the Materials Research Society.

Adams can be reached at Sandia National Laboratories, 1515 Eubank Blvd. SE, MS 1245, Albuquerque, NM, 87123 USA; tel. 505844-8317 and e-mail dpadams@sandia.gov.

Michael J. Aziz is the Gordon McKay Professor of Materials Science at Harvard University. Aziz has made significant contributions to the selforganization and kinetics of formation, thermal stability, and decay of nanoscale structures; thinfilm growth by pulsed laser melting and pulsed laser deposition; growth, diffusion, and viscous flow in stressed solids; and the kinetics of rapid alloy solidification.

Aziz is a recipient of the Presidential Young Investigator Award, the ONR Young Investigator Award, and the Sauveur Memorial Lectureship. He also is a fellow of APS and AAAS.

Aziz can be reached at the School of Engineering and Applied Sciences, Harvard University, 29 Oxford St., Cambridge,
MA 02138 USA; tel. 617495-9884 and e-mail maziz@harvard.edu.

Yongqi Fu is a research fellow and leader of the focused ion beam group in the Precision

Engineering and Nanotechnology (PEN) Center at Nanyang Technological University (NTU) in Singapore. He received his BEng (mechanical engineering, 1988), MSEng (optoelectronics, 1994), and $\mathrm{PhD}$ (optical engineering, 1996) degrees from Jilin University, Changchun University of Science and Technology, and the Chinese Academy of Sciences, respectively. Fu worked in the State Key Laboratory of Applied Optics from 1996 to 1998 as a postdoctoral fellow. He then worked in the PEN Center at NTU as a research fellow from 1998 to 2001. Fu also worked in the Innovation in Manufacturing Systems and Technology (IMST) Program of the Singapore-MIT Alliance as a research fellow from 2001 to 2005 . Fu is a guest professor at the Institute of Optics and Electronics, Chinese Academy of Sciences, and has authored more than 80 peer-reviewed journal papers and 21 conference proceeding papers.
Fu can be reached at Precision Engineering and Nanotechnology Center, School of Mechanical and Aerospace Engineering, Nanyang Technological University, 50 Nanyang Ave., Singapore 639798; tel. 65-67906336, fax 65-67904674, e-mail yqfu@ntu.edu.sg, and URL www.ntu.edu.sg/ home/yqfu/fyqpage.htm.

Lucille A. Giannuzzi is a product marketing engineer for focused ion beam and DualBeam ${ }^{\mathrm{TM}}$ systems at FEI Co. in Hillsboro, Oregon. She received her $\mathrm{BE}$ and MS degrees from SUNY-Stony Brook and her $\mathrm{PhD}$ degree from the Pennsylvania State University. Giannuzzi joined the University of Central Florida in 1994 and is founding director of the Materials

Characterization Facility. She joined FEI Co. in 2003.

Her research interests include ion-solid interactions, grain-boundary diffusion and segregation, and structure-property relationships of materials using ion and electron beam microscopy. She has received an NSF CAREER Award and is a member of numerous societies including the Materials Research Society. Giannuzzi has authored more than 100 publications, is an instructor at 


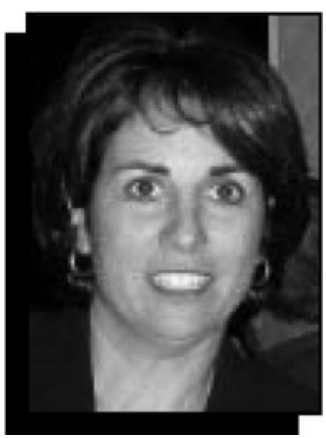

Lucille A. Giannuzzi

the Lehigh Microscopy

School, and is co-editor of the book Introduction to Focused Ion Beams.

Giannuzzi can be reached at FEI Co., 5350 NE Dawson Creek Dr. Hillsboro, OR 97124 USA; tel. 321-663-3806, fax 503726-7509, and

e-mail lucille.giannuzzi@ fei.com.

Jacques Gierak is responsible for focused ion beam research activity at

Laboratoire de

Photonique et de

Nanostructures (LPN)

in Marcoussis, France.

He graduated from the

Physics-Electronics

Department at

Conservatoire National

des Arts et Métiers in

Paris, where he earned

his DEA and PhD degrees

in instrumentation.

Gierak has been involved in FIB research since 1984 and was the coordinator of the European

Commission-funded

NanoFIB project. In 2004,

Gierak was awarded the

CNRS Crystal, a prize for exceptional engineering and technical achievement. He is the main author of several international CNRS patents related to ion sources, ion optics, and their control.

Gierak can be reached at Laboratoire de Photonique et de Nanostructures-CNRS, Route de Nozay, 91460 Marcoussis, France; tel.

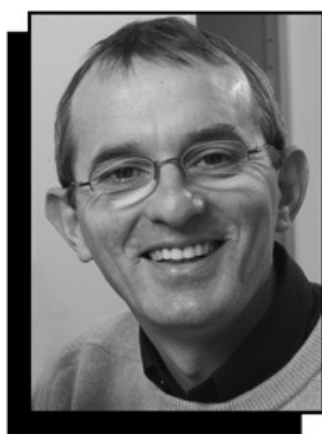

Jacques Gierak

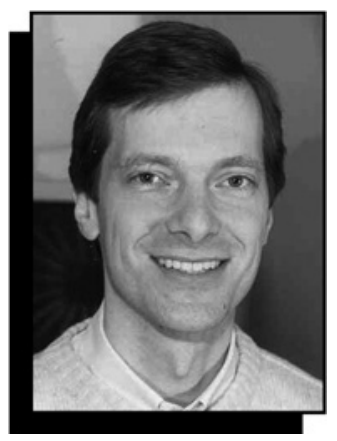

Gerhard Hobler

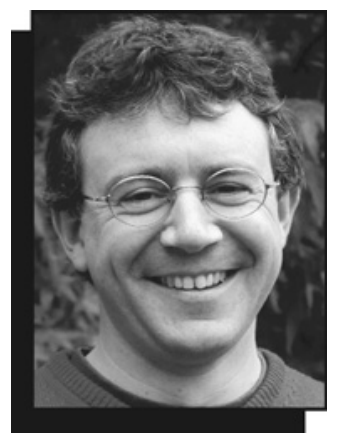

Lorenz Holzer

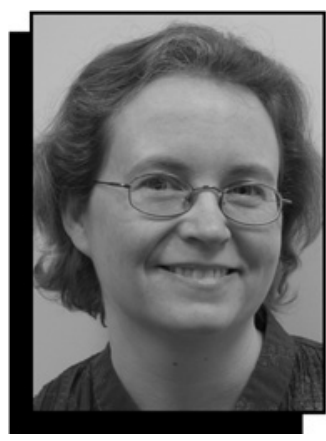

Beverley Inkson
33-1-69-63-60-75, fax 33-1-69-63-60-06, e-mail jacques. gierak@lpn.cnrs.fr, and URL www.lpn.cnrs.fr/fr/ PHYNANO/NanoFIB. php.

\section{Gerhard Hobler is an} associate professor of semiconductor electronics at the Vienna University of Technology. He received his Dipl-Ing, $\mathrm{PhD}$, and venia docendi degrees from Vienna University of Technology in 1985, 1988, and 1997, respectively. From 1996 to 1998, Hobler was a visiting scientist at Bell Laboratories, Lucent Technologies. His current research interests include atomistic modeling of ion-solid interactions and thermal processes following ion implantation. He has authored more than 100 papers in this field.

Hobler can be reached at the Institute of Solid State Electronics, Vienna University of Technology, Floragasse 7/362, A-1040 Vienna, Austria; tel. 43-1-58801-36233, fax 43-1-58801-36291, and e-mail gerhard.hobler@ tuwien.ac.at.

Lorenz Holzer is a senior scientist at Empa, the Federal Institute for Materials Testing and Research, in Dübendorf, Switzerland. He is leader of the 3D-Mat Group, which deals mainly with 3D microscopy and image-based modeling for quantitative microstructure analysis. For the last three years, he worked on FIB tomography methods for the topological characterization of complex granular textures and porous networks in cementitious and ceramic materials.

Holzer can be reached at 3D-Mat Group, Empa Materials Science and Technology, Dübendorf, Switzerland; tel. 41-44823-44-90, fax 41-44823-40-35, and e-mail lorenz.holzer@empa.ch.

Beverley Inkson is a senior lecturer and head of the NanoLAB group at the Department of Engineering Materials at the University of Sheffield. Inkson received her MA degree in physics and her $\mathrm{PhD}$ degree in materials science from the University of Cambridge. Her PhD studies were followed by an Alexander von Humboldt fellowship at the Max Planck Institute in Stuttgart, Germany, and a Royal Society research fellowship in nanomechanics at the University of Oxford. She is director of the U.K. NanoFIB Network and director of the RCUK Basic Technology Programme in Nanorobotics. Her current research interests are centered on the nanomanipulation and mechanical properties of nanostructured materials, including in situ FIB and TEM nanotesting, 3D tomography, NEMS, and nanowire physics.

Inkson can be reached at the Department of Engineering Materials, Mappin St., Sheffield, S1 3JD, United Kingdom; tel. 44-114-2225925, and e-mail beverley.

inkson@sheffield.ac.uk.

Takeo Kamino is technical advisor of the Naka Application Center at Hitachi High-

Technologies Corp. in Japan. He received his PhD degree in materials science and engineering at Ibaraki University in 1997. Kamino's current work focuses on the development of a technique for 3D structure observation of nanomaterials using a combination of FIB and high-resolution STEM or TEM. In addition, Kamino's research includes the development of TEM techniques allowing observation of atoms during sintering, reaction, and deposition. $\mathrm{He}$ has been a member of the board of directors of the Japanese Society of Microscopy since 2005.

Kamino can be reached at Naka Application Center, Hitachi High-
Technologies

Corporation, 11-1,

Ishikawa-cho,

Hitachinaka-shi, Ibarakiken, 312-0057, Japan; tel. 81-29-354-2970, fax 81-29-354-1971, and e-mail kamino-takeo@ nak.hitachi-hitec.com.

Joachim Mayer is a professor of physics at RWTH Aachen University, where he is head of the Central Facility for Electron Microscopy. He studied physics at the University of Stuttgart and received his PhD degree in 1988. Mayer spent two years as a postdoctoral researcher in the Materials Department at the University of California, Santa Barbara. Afterward, he returned to Max-Planck-Institut für Metallforschung in Stuttgart. In 1999, Mayer joined RWTH Aachen University. In addition to his work at RWTH

Aachen, Mayer is codirector of the newly founded Ernst Ruska Center for Microscopy and Spectroscopy with Electrons at the Research Center Jülich, Germany.

Mayer can be reached at the Central Facility for Electron Microscopy (GFE), RWTH Aachen University, Ahornstr. 55, D-52074 Aachen, Germany; tel. 49-241-8024345, fax 49-241-8022313, and e-mail mayer@ gfe.rwth-aachen.de. 


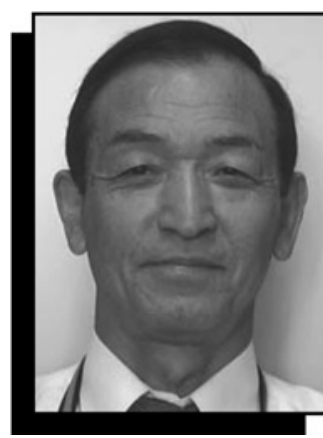

Takeo Kamino

Richard M. Langford works in the Materials Science Centre at the University of Manchester. He received his $\mathrm{PhD}$ degree from the Electrical Engineering Department at Imperial College. For the last decade,

Langford's main research interests have been in using focused ion beams for nanoengineering and nanofabrication and developing methods for sample preparation for electron microscopy.

Langford can be reached at the Materials Science Centre, University of Manchester,

Manchester, United

Kingdom; tel. 44-161-

3065914, fax 41-161-

3065912, and e-mail

richard.langford@

manchester.ac.uk.

Joseph Michael is a distinguished member of technical staff in the Materials and Process Sciences Center at Sandia National Laboratories. He received his BS, MS, and $\mathrm{PhD}$ degrees in materials science and engineering from Lehigh University. Before joining Sandia,

Michael was employed as a senior research engineer at Bethlehem Steel's Homer Research Laboratory. His research interests involve the application of advanced electron microscopies to the study of materials.

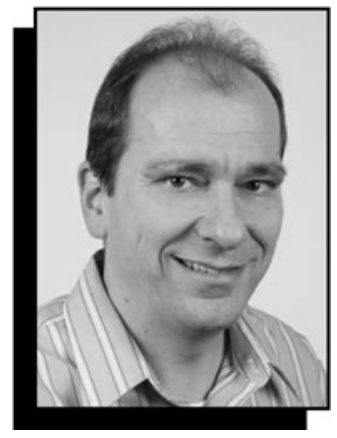

Joachim Mayer

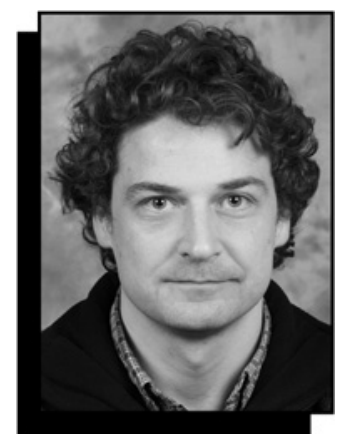

Richard M. Langford

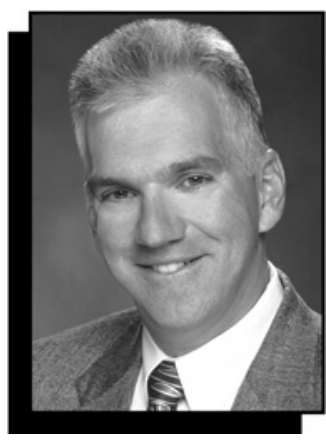

Joseph Michael

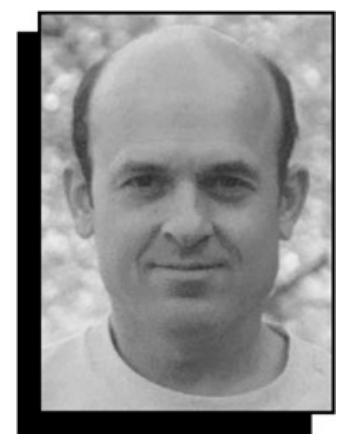

Warren J. MoberlyChan
Recently, Michael has been interested in the use of focused ion beam tools for the preparation of samples for electron backscatter diffraction, and for determining the $3 \mathrm{D}$ structure of materials. He has received the Burton Medal from the Microscopy Society of America and the Heinrich Award from the Microbeam Analysis Society. Michael also has published many papers on the application of electron microscopy to materials.

Michael can be reached at Sandia National Laboratories, MS 0886, PO Box 5800,

Albuquerque, NM 87185 USA; tel. 505-844-9115 and e-mail jrmicha@ sandia.gov.

\section{Warren J. MoberlyChan} is a microscopist at Lawrence Livermore National Laboratory in the Chemistry, Materials, and Life Sciences

Division. He received his $\mathrm{PhD}$ degree in materials science and engineering from Stanford University in 1991, and his ScB degree from Brown University. MoberlyChan has worked in the information storage industry at ReadRite, Komag, Quantum, MPI, and SSL. His research interests include thin-film interfaces and surfaces and the application of micro- scopy, FIB, and spectroscopy to study their chemical and mechanical properties.

MoberlyChan can be reached at Lawrence Livermore National Laboratory, MS L372, 7000 East Ave., Livermore, CA 94550-9234 USA; tel. 925-424-2721 and e-mail moberlychan2@1lnl.gov.

Paul Munroe is a professor of materials science and engineering and director of the Electron Microscope Unit at the University of New South Wales in Sydney, Australia. He received his $\mathrm{PhD}$ degree from the University of Birmingham in 1987, and for his thesis topic he characterized the microstructure of advanced titanium alloys. His principal research interests include structure-property relationships in thin-film and coated materials, and in the behavior of intermetallic alloys. Munroe received the CowleyMoodie Award from the Australian Society for Microscopy and Microanalysis for "outstanding physical sciences electron microscopy." He has published more than 160 journal papers and serves on the editorial board of Microscopy Research and Technique.
Munroe can be reached at the Department of Materials Science and Engineering, University of New South Wales, Sydney, NSW 2052 Australia; tel. 61-29385-4435 and e-mail p.munroe@unsw.edu.au.

Philipp M. Nellen works at RUAG Aerospace in Wallisellen, Switzerland. He received his $\mathrm{PhD}$ degree in physics from ETH Zurich, where he also worked in the Optics Laboratory at the Institute of Quantum Electronics on the subject of integrated optical chemo- and biosensors. Afterward, he joined Empa Switzerland. As a senior scientist at Empa, his interests started in the field of fiber-optical sensor systems for materials and structures, and the reliability of such systems. Following this research, he worked in the field of FIB micro- and nanostructuring of photonic and other devices.

The published work was done at Electronics / Metrology/Reliability Laboratory, Empa, Swiss Federal Laboratories for Materials Testing and Research, Überlandstrasse 129, CH-8600 Dübendorf, Switzerland. Nellen can be reached by e-mail at philipp.nellen@ruag.com.

Edward L. Principe is the focused ion beam applica- tions manager within the Nanotechnology Systems Division of Carl Zeiss SMT. He received his $\mathrm{PhD}$ degree in engineering science from the

Pennsylvania State University with research in the development of corrosion-resistant thin films. Before joining Carl Zeiss, Principe was a member of technical staff at Applied Materials, working on process development and materials characterization across a broad range of semiconductor processes. He also worked as a staff scientist at Charles Evans and Associates, specializing in XPS and Auger spectroscopy. His current work includes the development of 3D metrology, automated sample processing, and 3D nanofabrication.

Principe received the ISTA/EDFAS

Outstanding Paper Award in 2005 and the Microscopy and Microanalysis Best Paper Award in 2003. In addition, he has authored two text chapters on FIB applications and more than 20 journal publications.

Principe can be reached by e-mail at principe@smt.zeiss.com.

Thomas Schenkel is a staff scientist in the Accelerator and Fusion Research Division of 


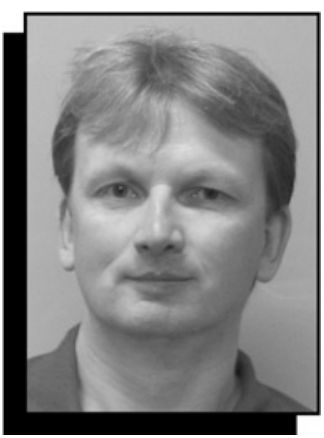

Paul Munroe

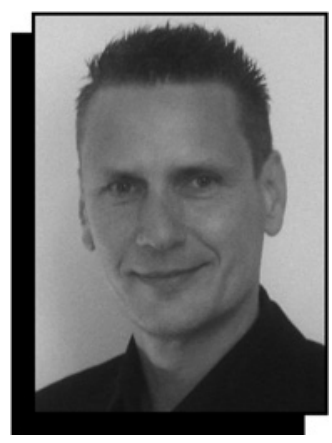

Philipp M. Nellen

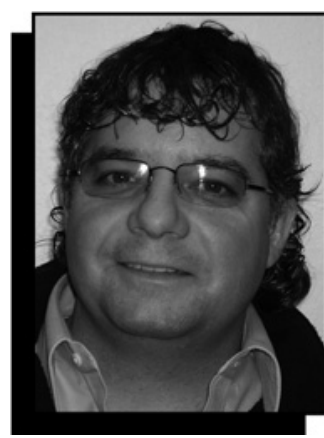

Edward L. Principe

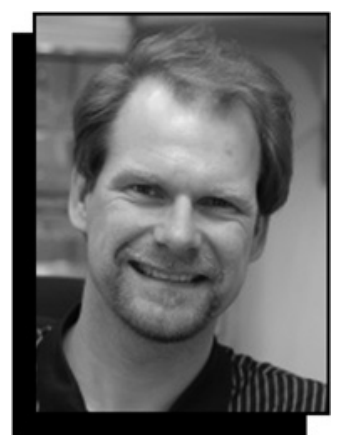

Thomas Schenkel

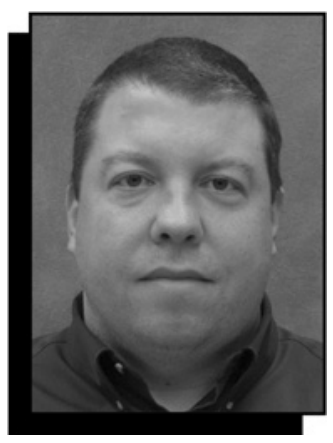

Michael D. Uchic
Lawrence Berkeley National Laboratory. He earned his $\mathrm{PhD}$ degree in physics from the Johann Wolfgang GoetheUniversity of Frankfurt am Main in 1997.

Following his postdoctoral research in the Chemistry and Materials Science Department at Lawrence Livermore National Laboratory,
Schenkel joined LBNL in 2000. His current research is focused on the development and testing of silicon-based quantum computer schemes.

Schenkel can be reached at Lawrence Berkeley National Laboratory, 1 Cyclotron Rd., 05R12, Berkeley, CA 94720 USA; and by e-mail at

t_schenkel@lbl.gov.

Michael D. Uchic is a materials research engineer in the Metals Development Group of the Air Force Research Laboratory, Materials and Manufacturing Directorate, at Wright Patterson Air Force Base. He received his
PhD degree from Stanford University, where he characterized the low-temperature mechanical properties of the intermetallic alloy $\mathrm{Ni}_{3} \mathrm{Al}$. Uchic started working with the Metals Development Group in 1998, and for the past five years his research efforts have focused on the develop- ment of new experimental methods to rapidly assess both the microstructure and mechanical properties of aerospace metals.

Uchic can be reached at AFRL/MLLM, 2230 10th St., Wright Patterson AFB, OH 45433 USA; tel. 937-255-4784 and e-mail michael.uchic@wpafb. af.mil.

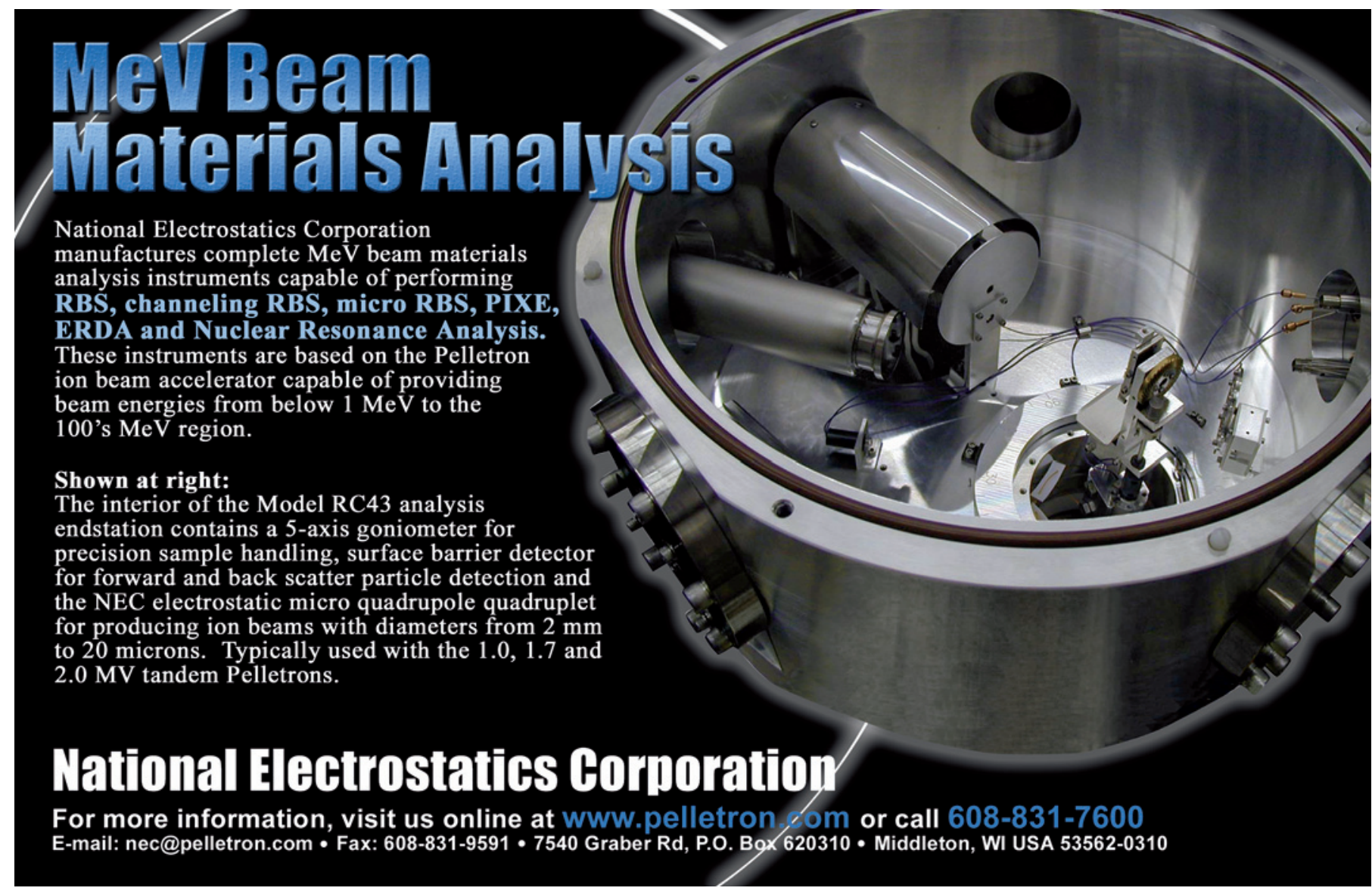

